\title{
Physics of Plasma Detachment in a Magnetic Nozzle
}

\author{
Christopher Deline ${ }^{1}$ \\ University of Michigan, Ann Arbor, MI 48109 \\ Greg Chavers ${ }^{2}$ \\ NASA Marshall Space Flight Center, Huntsville, AL 35805 \\ and Brian Gilchrist ${ }^{3}$ \\ University of Michigan, Ann Arbor, MI 48109
}

\begin{abstract}
The demonstration of plasma detachment from an applied magnetic field is of paramount importance to many future space propulsion designs. Ion engines and Hall thrusters do not produce a magnetized plasma, so the question of plasma magnetization has never been raised for these technologies. Several future propulsion technologies, including helicon plasma supplies, do produce a highly magnetized plasma that may or may not break from the applied magnetic field. It has been theorized that a sufficiently energetic plasma flow will detach from applied magnetic nozzle fields by stretching the magnetic field lines away from the plasma source. Experiments are being conducted at the NASA Marshall Space Flight Center intending to study this type of magnetic detachment. This paper will present preliminary results from that experiment, specifically density measurements that indicate a possibility of detachment occurring in this experiment.
\end{abstract}

\section{Nomenclature}

$\begin{array}{ll}B & =\text { Magnetic field intensity } \\ m_{i} & =\text { Ion mass } \\ \mu_{0} & =\text { Permeability of free space } \\ n_{e} & =\text { Density of electrons } \\ n_{i} & =\text { Density of ions } \\ q & =\text { Electron charge } \\ r_{p} & =\text { Radius of Langmuir probe }\end{array}$

$\begin{array}{ll}T_{e} & =\text { Electron temperature } \\ v & =\text { Plasma directed flow velocity } \\ v_{A} & =\text { Alfvén velocity } \\ \Omega_{c i} & =\text { Ion cyclotron frequency } \\ y & =\text { Vertical coordinate in experiment } \\ \hat{z} & =\text { Axial coordinate in experiment }\end{array}$

\section{Introduction}

EVERAL Electric Propulsion (EP) systems have been proposed with high specific impulse ( $\mathrm{I}_{\text {sp }}$ ) and high power $\checkmark$ for future exploration missions. ${ }^{1,2,3}$ These propulsion systems utilize a plasma source aligned coaxially with a number of high strength $(0.1 \mathrm{~T})$ magnets, which shape and confine the plasma as it travels down the axis of the rocket. One such propulsion system is the VAriable Specific Impulse Magnetoplasma Rocket (VASIMR) ${ }^{4}$, in which the plasma is also heated by a secondary RF power system and antenna that couple power to the plasma at the ion cyclotron frequency $\Omega_{c i}=q B / m_{i}$. The axial flow velocity $v$ of the plasma is further increased by the diverging magnetic field at the aft end of the rocket, due to conservation of magnetic moment. This configuration forms a

\footnotetext{
${ }^{1}$ Graduate Student, AIAA Student Member cdeline@umich.edu

${ }^{2}$ Physicist, NASA/MSFC/ER24

${ }^{3}$ Professor, Electrical Engineering and Space Systems, AIAA Associate Fellow
} 
magnetic nozzle, which directs the plasma exhaust aft of the spacecraft without exposing material surfaces to the highly energetic exhaust plasma.

It must be shown both in theory and in experiment that at a certain point downstream of the rocket, the plasma flow continues in the axial direction, detached from the influence of applied magnetic fields. At a practical level, this means that the plasma exhaust is no longer affected by the magnetic field supplied by the spacecraft. This does not require local magnetic field to go to zero however. In the presence of plasma current loops, a self-produced magnetic field may still exist in the plasma which reduces but does not eliminate the local magnetic field. In this way, the lines of constant magnetic flux are able to stretch to infinity along with the plasma particles, akin to how the solar wind carries the interstellar magnetic field to the edges of the solar system. This "frozen-in" field condition is achieved when the flow speed $v$ reaches the Alfvén velocity $v_{A}=B / \sqrt{\mu_{0} n_{i} m_{i}}$.

A particular form of magnetic nozzle has been proposed ${ }^{5}$ consisting of straight diverging magnetic field lines created by external coil magnets. This topology is expected to minimize the occurrence of plasma current loops which may produce nozzle inefficiencies and instability. So long as any plasma current arises after the flow reaches the Alfvén velocity, its effects can not be transferred back to the rocket. A smooth transition from sub- to superAlfvénic flow is thus desired within the nozzle.

An experiment is being performed at the NASA Marshall Space Flight Center, Huntsville AL which has implemented this style of magnetic nozzle ${ }^{6}$. Instruments are being used to characterize the plasma flow along the length of the plasma flow, particularly at the nozzle aperture where the super-Alfvénic transition is expected to occur. Velocity, magnetic field and density data are being gathered as part of this experiment. In this paper, a preliminary analysis of density measurements at several axial locations is presented.

\section{Laboratory Experiments}

The creation of laboratory conditions suitable for magnetic detachment is governed by several conditions. First, the experiment must employ a plasma source capable of producing a sufficiently dense and energetic flow to reach the Alfvén velocity. Second, the vacuum chamber used must be large enough to allow for the long pathlengths required to achieve super-Alfvénic flow $\left(v>v_{A}\right)$. Third, the vacuum chamber must be kept at a neutral background pressure sufficiently low to keep ion-neutral charge-exchange mean free path less than the path length for achieving $\mathrm{v}>v_{A}$.

One example of such a favorable experiment setup is the NASA Marshall Detachment Demonstration Experiment (DDEX) in which a large $\left(30 \mathrm{~m}^{3}\right)$ vacuum chamber achieves the above conditions suitable for detachment research. (see Fig. 1 and Reference 6) The chamber uses current carrying coils to produce a small divergence angle magnetic nozzle with straight field lines. Two diffusion pumps allow the use of lighter gases (primarily helium) and keep the chamber below 10 microTorr resulting in an estimated $10 \mathrm{~m}$ charge exchange mean free path. The chamber

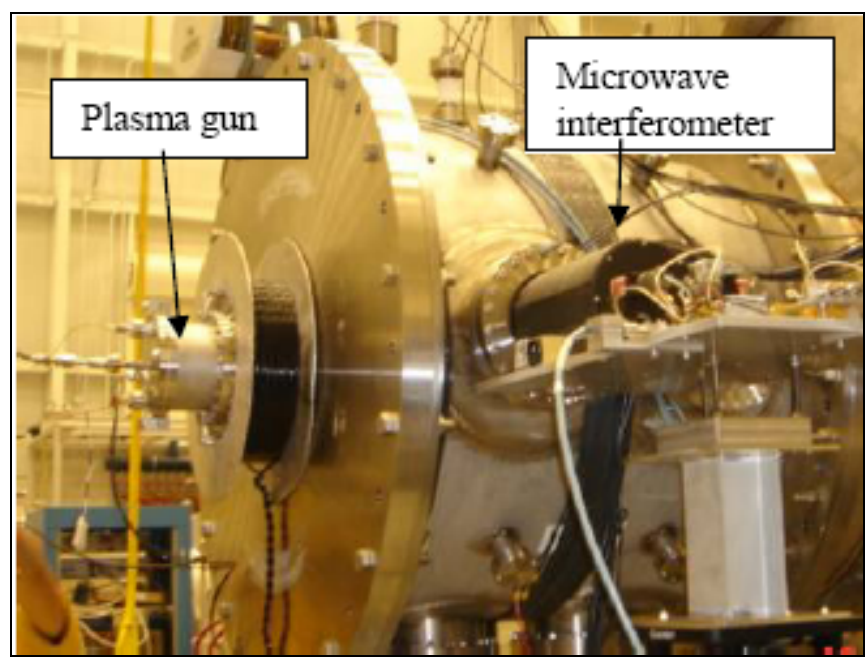

Figure 1: Detachment Demonstration Experiment (DDEX) magnetic nozzle section. Shown are plasma gun, $R F$ interferometer and magnetic nozzle coils. Not shown: $30 \mathrm{~m}^{3}$ expansion chamber and vacuum pumps coordinate axis is arranged with axial position $z=0 \mathrm{~m}$ denoting the exit aperture of the magnetic nozzle.

External current carrying coils produce a peak magnetic field intensity of $700 \mathrm{G}$ on-axis, falling off with $1 / Z^{2}$ until $Z=1 \mathrm{~m}$ where the applied field intensity becomes $\sim 1 \mathrm{G}$. Multiple large-diameter nozzle coil magnets are used in conjunction with a small bore, high-field choke magnet to produce straight diverging magnetic field lines. At $z=1 \mathrm{~m}$, superposition of the earth's field $\left(0.5 \mathrm{G}, 60^{\circ}\right.$ inclination) with the applied field results in constant flux lines bending downwards into the chamber walls as shown in Fig 2. Magnetometer measurements on-axis confirm this field map.

For some of the tests, the large-diameter nozzle coil magnets are used to adjust the magnetic field downstream of the thruster, producing a magnetic cusp configuration (Fig. 3). This configuration is achieved by running

American Institute of Aeronautics and Astronautics 
reverse current through the coils, resulting in zero field on-axis by $Z=0 \mathrm{~m}$. According to theory, this field configuration will perturb the density of a magnetically attached plasma, but will not perturb the density of a magnetically detached plasma.

For the data set considered here, one plasma source is used - a pulsed DC plasma washer gun operated at $300 \mathrm{~kW}$. Data is presented using helium as a feedgas, although other gases (hydrogen, argon) are possible. Typical pulse lengths are a few milliseconds, with plasma densities reported at $n_{e} \approx 1 \times 10^{14} \mathrm{~cm}^{-3}$ and electron temperature $T_{e} \approx 10$ $20 \mathrm{eV}$ at the source aperture ${ }^{7}$. Given these temperatures, and a magnetic nozzle two meters in length, the transit time is $0.1 \mathrm{~ms}$ for helium. The pulse length is an order of magnitude longer than the transit time, thus allowing the plasma to reach steady state during this experiment.

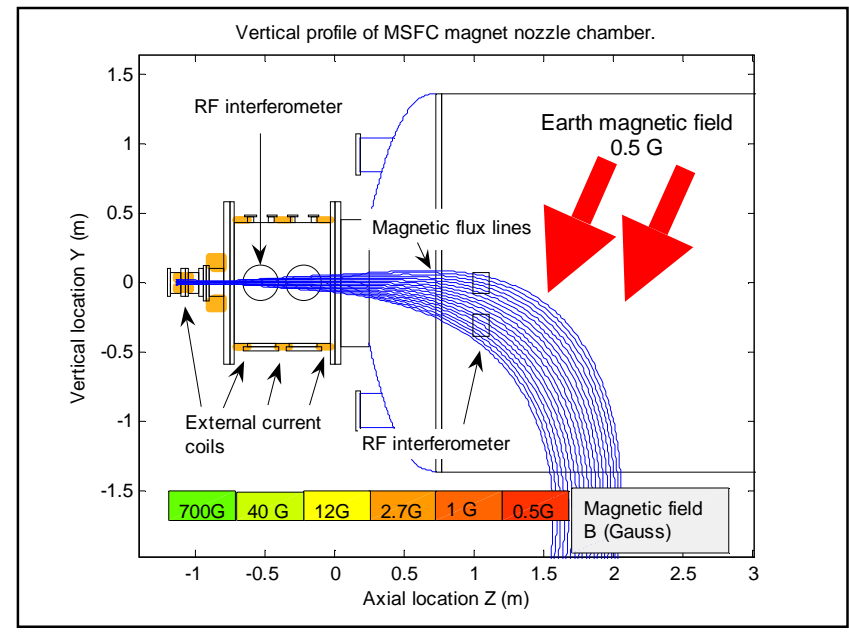

Figure 2. Normal magnetic field profile in the experiment. Trajectories are lines of constant magnetic flux mapped back to the plasma gun source. Coil Ampturns are as follows: 11000, 1200, 420 and 300. Magnetic field map confirmed by magnetometer measurements.

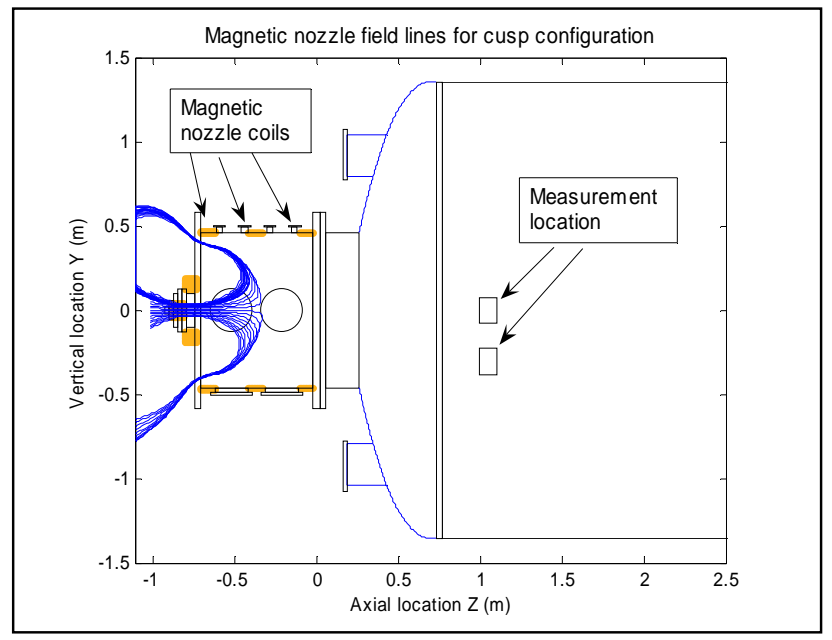

Figure 3. Magnetic cusp configuration. Trajectories are lines of constant magnetic flux, mapped back to the plasma source. This configuration is used to perturb density measurement of a magnetized plasma. No effect is encountered for an unmagnetized exhaust. Coil Amp-turns: $11000,-1200,-420$ and -300

\section{Measurements}

An X-Z position table inside the chamber allows radial scans of the plasma exhaust. A Langmuir triple-probe ${ }^{8}$ is positioned on the end of a boom, with a cylindrical probe-tip radius of $0.076 \mathrm{~cm}$. This small Langmuir probe is oriented perpendicular to the plasma flow and allows fine spatial resolution scanning of the plume. A 6-element Langmuir triple-probe array is mounted vertically on the boom to determine the plume's radial distribution for each shot. The tip radius for these larger probes is $0.12 \mathrm{~cm}$. Other instruments are mounted on the $\mathrm{X}-\mathrm{Z}$ position boom, which are not considered in this paper. These instruments (Faraday probe, B-dot probes) are positioned hundreds of Debye lengths away from the Langmuir probes, and will not affect their readings. Additional chamber diagnostics include microwave interferometers which are positioned at three locations to provide spatial and temporal density measurements. A polychromatic quadrature interferometer is used which operates simultaneously at $70 \mathrm{GHz}, 90$ $\mathrm{GHz}$ and $110 \mathrm{GHz} .{ }^{9}$ This instrument is positioned near the nozzle entrance, about $0.5 \mathrm{~m}$ downstream from the plasma source. The spatial resolution for this interferometer is typically $0.015 \mathrm{~m}$, with a minimum sensitivity of $10^{16} \mathrm{~m}^{-3}$ over the $0.1 \mathrm{~m}$ pathlength. Upstream plasma density measurements are taken via Langmuir probe along the chord of this interferometer. A second interferometer instrument is located $1.5 \mathrm{~m}$ downstream at the nozzle exit aperture. This $15 \mathrm{GHz}$ quadrature interferometer ${ }^{10}$ provides a measurement resolution of at least $n_{e}=10^{15} \mathrm{~m}^{-2}$ for line-integrated measurements along the $1.7 \mathrm{~m}$ beam length. The cutoff density (peak density) of the interferometer is $n_{e}=3 \times 10^{17} \mathrm{~m}^{-3}$. Two independent interferometer chords are positioned at axial location $z=1.12 \mathrm{~m}$, with one chord on the axial centerline, and one positioned below it, at $y=-0.3 \mathrm{~m}$. Downstream plasma density measurements are taken via Langmuir probe along the centerline chord of this interferometer.

Accurate plasma density plots are produced by radially scanning the small Langmuir triple-probe across the plasma column along a microwave interferometer chord. Shot-to-shot variation is factored out by normalizing each probe data point to the interferometer measurement for that shot. Langmuir probe measurements at various radial positions provide the radial shape of the plasma column. The interferometer chord density measurement is matched 
to the line integrated probe density measurement by introducing a scalar constant. This takes advantage of the benefits of both instruments: accurate non-intrusive density measurements from an RF interferometer, and high spatial resolution measurements from an electrostatic probe. The radial profile of the plasma is thus accurately measured over several plasma shots and probe measurements.

Errors may be introduced in the Langmuir probe measurements due to large Debye lengths with respect to the probe radius $r_{p}$. This is only the case for downstream measurements where the thin-sheath assumption is invalid, or plasma Debye length is greater than $10 \%$ of the probe radius. While the absolute density measurement will not be affected much (as this is determined by microwave interferometer measurement) this thin-sheath violation will introduce a 5-10\% error term in the calculated plasma column diameter for downstream measurements.

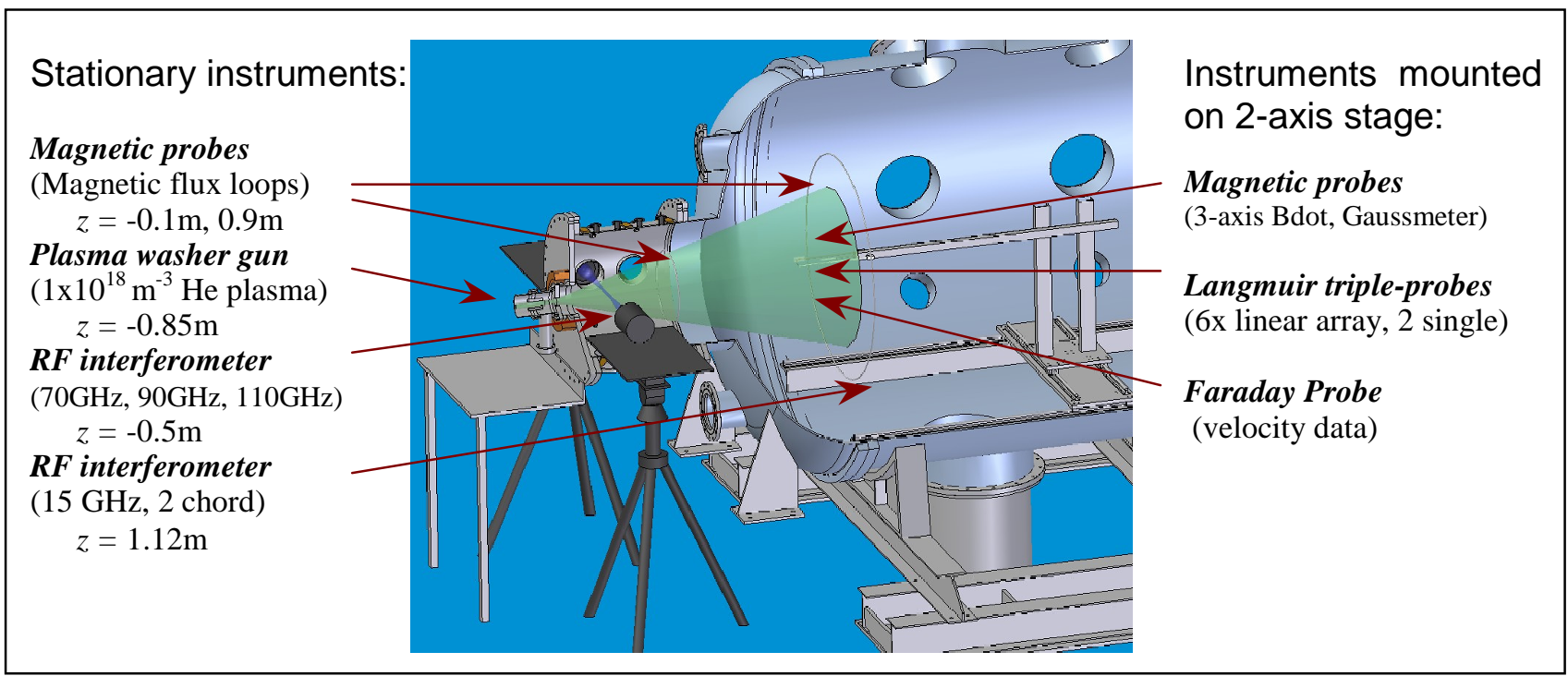

Figure 4. Diagnostic setup of the MSFC magnetic nozzle experiment. Axial location of diagnostic instruments are shown. Coordinates as follows: r-axis is transverse to the plasma flow in the horizontal direction. Z-axis is parallel to plasma flow down the chamber. Y-axis is along a vertical cross-section of the chamber.

\section{Preliminary Experimental Data}

Density profiles were measured for a helium plasma plume produced by the stacked-washer gun. A Gaussian density profile centered on the axis was recorded both upstream (Fig. 6) and downstream (Fig. 7). A peak electron density of $n_{e}=8 \times 10^{18} \mathrm{~m}^{-3}$ was recorded upstream and $n_{e}=2.7 \times 10^{15} \mathrm{~m}^{-3}$ was recorded downstream. Based on the full width half max profile measurement, the plasma column expanded from a $14 \mathrm{~cm}$ diameter at the nozzle entrance to $82 \mathrm{~cm}$ at the nozzle exit $1.62 \mathrm{~m}$ downstream. Both measurements suggest that an axisymmetric exhaust is continuing downstream without being perturbed by the curved magnetic field. Total plasma load (total number of particles released per plasma shot) was calculated by spatial integration of electron flux over the (quasineutral) plasma plume and time integration over the shot duration. At the upstream interferometer location $(\mathrm{z}=-0.5 \mathrm{~m})$ the total electron load per shot was $3.4 \times 10^{18}$ electrons. At the downstream interferometer location $(\mathrm{z}=1.12 \mathrm{~m})$ the total electron load per shot was $2.1 \times 10^{18}$ electrons. A total neutral load of $2.9 \times 10^{19}$ neutral particles was calculated from a pressure rise of $3 \times 10^{-5}$ Torr in the $30 \mathrm{~m}^{3}$ chamber. The total ionization fraction was $12 \%$ with $62 \%$ of the plasma flux being recorded downstream. Given errors associated with assuming an axisymmetric plasma plume, and particle collisions with probe diagnostics, this result can be taken to mean that a majority of the plasma exhaust is arriving downstream, i.e. following the chamber axis.

Velocity measurements in this experiment remain too imprecise to state whether the local plasma velocity is increasing or decreasing throughout the plasma shot. Time-of-flight correlation between several photomultipliers and flux loops suggest a bulk flow velocity of $5-15 \mathrm{~km} / \mathrm{s}$. However, this estimate has not been refined enough to report the Alfvén Mach number at any specific location with a high degree of precision. 


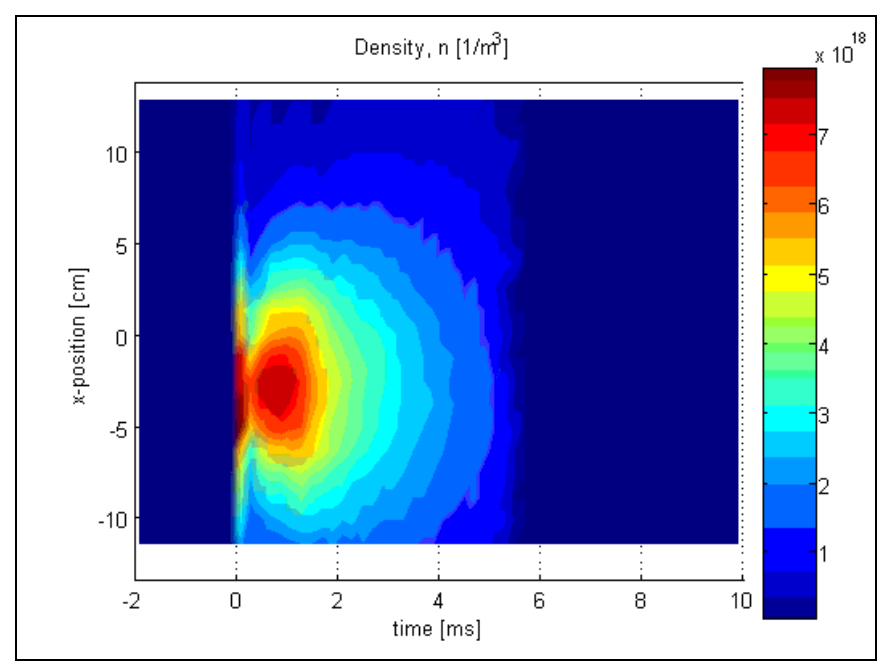

Figure 5: Upstream density measurement. Peak $n_{e}=$ $8 \times 10^{18} \mathrm{~m}^{-3}$. Plasma profile is a Gaussian distribution with width $=0.14 \mathrm{~m}$ full width half $\max (F W H M)$. Measurement location is $Z=-0.5 m$ and was taken during standard magnetic field configuration.

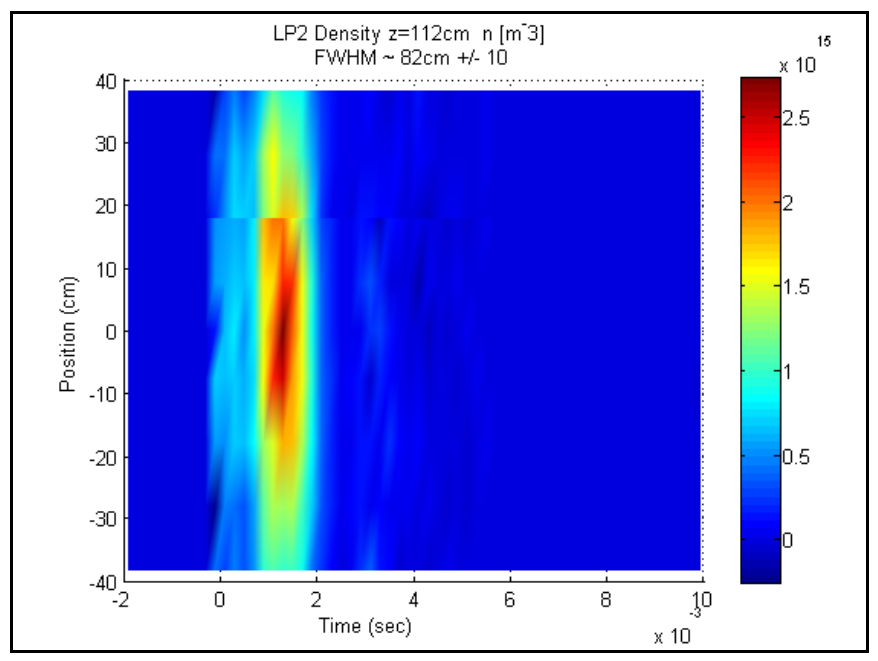

Figure 6: Downstream density measurement. Peak $n_{e}=$ $2.7 \times 10^{15} \mathrm{~m}^{-3}$. Plasma profile is a Gaussian distribution with diameter $=0.82 \mathrm{~m}$ FWHM. Measurement location is $Z=$ 1.12 m.and was taken during standard magnetic field configuration.
An attempt was made to perturb the plasma flow by modulating the magnetic fields in the nozzle. The strongly diverging magnetic flux lines seen in Fig. 3 were produced by running reverse current through the nozzle magnet coils. This configuration was termed the cusp field configuration. When compared with the standard magnetic configuration of Fig. 2, the cusp field configuration produced a very strongly diverging magnetic field, with zero field strength on the axis after the nozzle aperture. The result of this curving field was that no appreciable change was detected in plasma density, and the radial profile remains essentially as shown in Fig. 6. A side by side comparison of two separate shots is shown in Fig. 7 taken by the downstream $15 \mathrm{GHz}$ interferometer. The result is that little difference besides normal shot-shot variation exists between the standard magnetic configuration and the cusp field configuration. This leads to the conclusion that beyond the magnetic nozzle aperture at $z=0$ the plasma density is not being influenced much by the applied magnetic field. Velocity measurements in this area remain too inconclusive to state whether momentum is also being carried downstream, or if a slowing of the plasma flow is resulting from magnetic field modulation. However, the density measurements combined with total electron flux measurements do point to a possibility of plasma detaching from applied magnetic field lines in the exhaust region of this experiment.

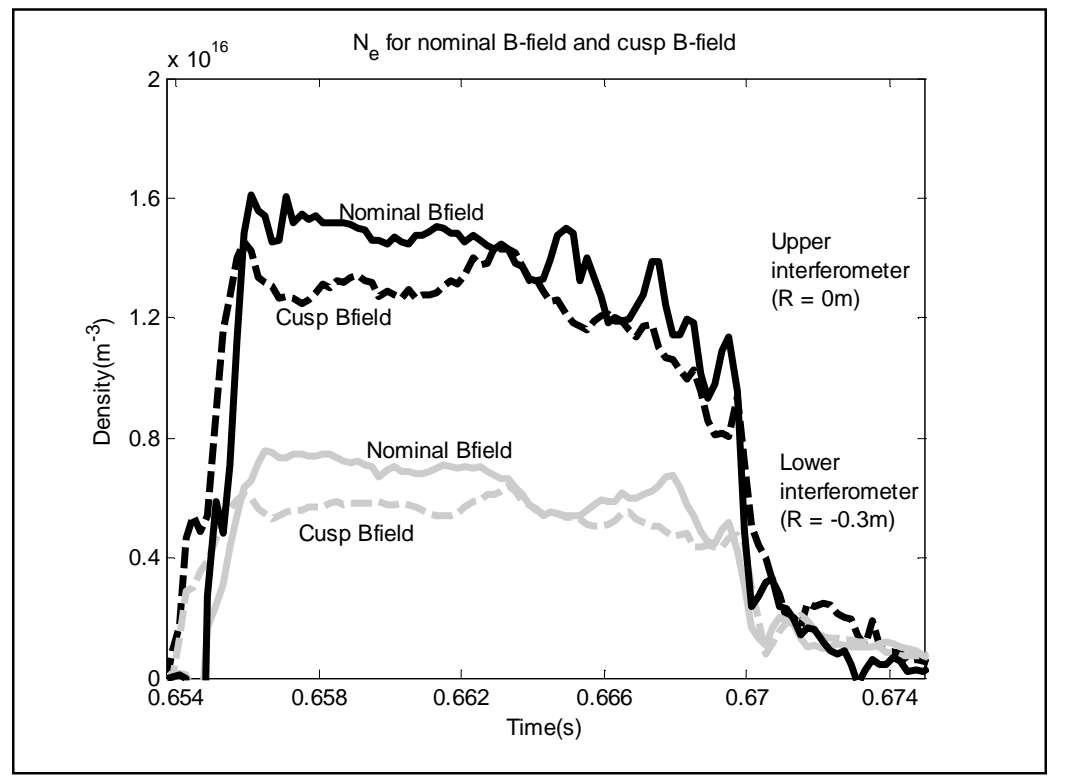

Figure 7: Comparison of density with nominal magnetic nozzle, and cusp magnetic nozzle. Measurements taken at $z=1.1 \mathrm{~m}$ with 2-chord interferometer. Magnetic field configuration shown in Fig. 4 and Fig. 5. No appreciable difference in plasma flow under different magnetic field conditions. 


\section{Conclusions / Future Work}

Although the data analysis for this experiment is not yet complete, several preliminary results are evident. Under all conditions of the nozzle magnets tested (including a reverse-field, cusp configuration), the downstream plasma density approximately followed a Gaussian profile centered on the axis. Also, the total plasma load measurement suggests that plasma is continuing down the axis of the rocket despite curved magnetic fields. This is a promising result for an eventual case for magnetic detachment.

However, several pieces of the puzzle are still needed to make a strong argument that magnetic detachment is indeed occurring in this experiment. These include more accurate velocity measurements and some solid B-dot probe evidence. It is also important to make direct observations of plasma flow diverting from the chamber axis to follow transverse field lines, as reported in earlier experiments ${ }^{11}$. This would show a condition under which the plasma remains attached to magnetic field lines, and prove the magnetized nature of the plasma at its source. In addition, more complete measurements will be required to diagnose some aspects of the super-Alfvénic transition, including accurate measurement of local magnetic field intensity downstream, and precise determination of how the magnetic transport correlates with Alfvén velocity.

One challenging aspect of this experimental work is the collection of reliable B-dot probe data and velocity measurements. Since the measurement of the local Alfvén Mach number $M_{A}$ requires both density velocity and magnetic field information, it is crucial to obtain this data with a high degree of precision. Magnetic field measurements in particular may prove to be difficult because field line stretching is only expected to change the total magnetic flux at any given point by about $10 \%$. The magnetic nozzle aperture is already far enough downstream to have a much reduced magnetic field, so a change of less than 1 Gauss is all that is expected through the process of detachment. Future work includes creating magnetic probes capable of detecting this change in magnetic field, without becoming overwhelmed by the noisy RF plasma environment. Also, further work must be done to refine velocity and density analysis for the data already collected.

\section{Acknowledgments}

This work was supported by NASA Marshall Space Flight Center, a NASA BAA contract awarded to the University of Texas and a NASA GSRP fellowship (Chris Deline). Special thanks to Boris Breizman (UT-Austin), for consultation on theory, and Jonathan Jones and Chris Dobson (MSFC) for help with data reduction and analysis.

\section{References}

${ }^{1}$ F.R. Chang Diaz, "Research Status of the Variable Specific Impulse Magnetoplasma Rocket”, Fusion Technology 35 (1T) pp. 87-93, 1999

${ }^{2}$ C. Charles, R.W. Boswell, "Current-Free Double-Layer Formation in a High-Density Helicon Discharge,” Applied Physics Letters, 82 (9) 2003

${ }^{3}$ J.Gilland, "Application of a Helicon Discharge to Electric Propulsion" $34^{\text {th }}$ AIAA Joint Propulsion Conference, paper 3934 , 1998

${ }^{4}$ A.Arefiev, B.Breizman, "Theoretical Components of the VASIMR propulsion concept" Physics of Plasmas 11 (5) 2004

${ }^{5}$ A.V. Arefiev, B.N. Breizman, "Magnetohydrodynamic Scenario of Plasma Detachment in a Magnetic Nozzle" Physics of Plasmas 12 (4) 2005

${ }^{6}$ G. Chavers et al., "Status of magnetic nozzle and plasma detachment experiment", AIAA Space Technology and Applications International Forum, February 2006

7 G. Fiksel, A.F. Almagri, D. Craig, M. Lida, S.C. Prager, J.S. Sarff, "High current plasma electron emitter" Plasma sources science and technology 5 pp. 78-83 (1996)

${ }^{8} \mathrm{~S}$. Buffton, "Exit plane plasma measurements of a low-power hydrazine arcjet", PhD Thesis University of Illinois, UrbanaChampaign 1996

${ }^{9}$ C. Dobson, J. Jones, D.G. Chavers, "Instrument reflections and scene amplitude modulation in a polychromatic microwave quadrature interferometer" Review of Scientific Instruments 75 (3) 2004

${ }^{10}$ B. Gilchrist, S. Ohler, A. Gallimore, "Flexible microwave system to measure the electron number density and quantify the communications impact of electric thruster plasma plumes", Review of Scientific Instruments 68 (2) 1997

${ }^{11}$ F. Wessel, S. Robertson, "Polarization of an intense space-charge-neutral ion beam incident upon a magnetic field", Physics of Fluids 24 (4) 1981 